\title{
Wide-Area Power Oscillation Damping Control (POD) in Nordic Equivalent System
}

Nilanjan Ray Chaudhuri, Imperial College London

Alexander Domahidi, ETH Zurich

Rajat Majumder, ABB Corporate Research, Vasteras

Balarko Chaudhuri, Imperial College London

Petr Korba, ABB Switzerland Ltd., Baden-Daetwill

Swakshar Ray, ABB Corporate Research, Vasteras

Kjetil Uhlen, Sintef, Trondheim 


\begin{abstract}
A study is presented on power oscillation damping control (POD) using wide area measurements applied to a single static var compensator (SVC). An equivalent power system model representing key characteristics of the Nordic power system is used. Feedback signals from remote phasor measurment units (PMUs) in Norway and Finland are used to damp the critical inter-area modes through a large SVC unit located in south-east Norway. A comparison between two control design approaches - (i) model based POD (MBPOD) - dependant on accurate system model and (ii) indirect adaptive POD (IAPOD) - which relies only on measurements - is made. For MBPOD an optimization approach is used to obtain the parameters of the controller while the IAPOD is based on online Kalman filter estimation and adaptive pole-shifting control. It is shown that the IAPOD yields almost similar performance as the MBPOD with very little prior information about the system. The performance comparison is verified for several tie-line outages.
\end{abstract}

\title{
1 Introduction
}

Use of wide-area (remote) signals improves the effectiveness of Power Oscillation Damping Control (POD) $[1,2,3,4,5]$. With technological advancements and increasing deployment of wide-area monitoring infrastructure, closed-loop damping control employing wide-area signals is a definite possibility. Classical control theory has been and continues to be adopted for design of damping controllers which require a reasonably accurate model of the system at a particular (nominal) operating condition [6]. However, lack of availability of accurate and updated information about each and every dynamic component of a large inter-connected system and its ever changing nature often puts a fundamental limitation on such model based control design approaches.

Indirect adaptive controllers (also referred as self-tuning controllers in literature), which rely solely on system measurements, were proposed for power system stabilizers (PSS) $[7,8]$ in the early eighties and also for the FACTS devices $[9,10,11]$ and HVDC [12] in recent past. These controllers have fixed structures but their parameters are updated online based 
on the estimated model of the system and thus can adapt to the changes in operating conditions. Recursive least squares (RLS) method is usually used for estimation along with pole-shifting, minimum variance techniques to derive the control action at each sampling interval [8]. Moreover, in digital implementation it is possible and sometimes recommended to apply different sampling rates for the estimator and the controller $[13,14]$.

Indirect adaptive controllers are attractive as they do not rely on accurate and updated system models which is increasingly becoming difficult to obtain. They also adapt to changing operating conditions and do not need extensive re-tuning. In this paper a case study is presented to compare the performance of a model based POD (MBPOD) and an indirect adaptive POD (IAPOD) in the context of the Nordic equivalent system. The performance criteria is satisfactory damping of the two critical inter-area modes under different scenarios (e.g. tie-line outages).

In this exercise the parameters of the MBPOD are optimized for desired performance with optimal control effort under different operating conditions. For the IAPOD, Kalman filtering [9] and self-optimizing pole shifting [15] approaches are used unlike online prony analysis [12]. Linear analysis and non-liner simulation results show that the IAPOD is able to produce almost similar performance as the MBPOD, but with very little prior knowledge about the system. Besides the nominal condition, several tie-line outages in the Nordic equivalent system were considered to substantiate these claims.

The rest of the paper is organized as follows. Section II gives an overview of the Nordic equivalent system in terms of its modal behavior and highlights the selection of wide-area feedback signals. Constrained optimization framework for the MBPOD is described in Section III along with the controller parameters obtained and used in this study. In Section IV, the IAPOD approach is presented along with a list of parameter values used here. Section V and VI contains the results from linear analysis with both MBPOD and IAPOD and a comparison with non-linear simulations, respectively. 


\section{Overview of Nordic Equivalent System}

A reduced equivalent of the Nordic system is used in this case study. The detailed model with approximately 3000 buses, 4000 branches and 1100 generators [16] is reduced down to an equivalent 20 generator, 36 bus system, see Fig. 1. The aim is to retain the modal behavior of the two most critical poorly damped inter-area modes. A static VAr compensator (SVC) is included in the model at Hasle substation in south-east Norway as in practice. The total number of state variables for the reduced equivalent is 296 .

There are two critical inter-area modes, $0.29 \mathrm{~Hz}$ and $0.55 \mathrm{~Hz}$ with $4.8 \%$ and $5.4 \%$ damping, respectively under nominal condition. The first mode $(0.29 \mathrm{~Hz})$ comprises of the Finnish generators swinging against the rest while the second mode $(0.55 \mathrm{~Hz})$ involves the generators towards the north of Finland, Sweden and Norway swinging against those in the southern parts of these countries. The geographical distribution of the modeshapes is shown in Fig. 2.

Following critical contingencies like outage of one of the parallel lines connecting i) 65006700 (Norwegian coast line), ii) 7100-7000 (Finnish line) or iii) 3359-5101 (Hasle line), the damping of the inter-area modes could be as low as $2-3 \%$, see Table 1, requiring improvement through supplementary control. Here the challenge is to ensure satisfactory damping of both the modes by designing a POD for the SVC at Hasle.

With a number of PMUs (four in Norway and two in Finland are considered for this study) installed throughout the Nordic system, remote feedback signals were used instead of local ones to exploit the benefits mentioned in $[1,2,3,4]$. Both the magnitude and the phase angle of the residues were considered for selecting the appropriate feedback signals for reasons described in [17]. Only the difference between voltage angles available from the PMUs were chosen as potential candidates [16]. The magnitude and phase angle of the residues of the candidates are summarized in Table 2 . Voltage angle difference between the PMUs at 6100 (at Nedre Rossaga) and 7000 (in southern Finland) has high residue magnitude for both modes and the phase angles are also in the same direction unlike 6700-7000 which are in opposite directions. Hence, 6100-7000 was selected as the most appropriate signal for the POD. 


\section{Model Based POD (MBPOD)}

The general optimization framework for designing a MBPOD is presented here, see [18] for more details. For $n$ critical inter-area modes which are highly observable from $n$ different locations the controller is made up of $n$ channels with a gain $\left(K_{1}, K_{2}, \ldots K_{n}\right)$ and a maximum of three first order lead-lag blocks in each channel, see Fig. 3. Also the standard low pass noise filters $\left(T_{m}=0.01 \mathrm{~s}\right)$ and the high-pass washout blocks $\left(T_{w}=10.0 \mathrm{~s}\right)$ are present in each channel. Measured signals are denoted as $P_{1}, P_{2}, \ldots P_{n}$ and the control input to the FACTS device as $u$ in Fig. 3. The structure and order of the compensators in each channel are chosen to resemble one of the standard power system stabilizer structures described in [19].

\subsection{Objective Function}

The controller is required to ensure that oscillations settle within $10.0 \mathrm{~s}$ for all the operating conditions considered during the design with optimum control effort. Determination of the gain and time constants of the controller, required to achieve the above specifications, can be formulated as a multi-objective optimization problem and solved using any standard optimization technique.

The design specifications are as follows:

1. Modal oscillation due to each critical inter-area mode should settle within $10.0 \mathrm{~s}$ for all the operating conditions considered during design.

2. Frequency of oscillation of the inter-area modes should not change appreciably from their open-loop values.

3. Control effort should be optimum.

For individual modal oscillations, which closely exhibits a second-order system behavior, settling time $\left(T_{s}\right)$ depends on the product of the damping ratio $(\zeta)$ and the natural frequency of oscillation $(\omega)$, which in turn is decided by the real part $(\sigma)$ of the poles [6]. Hence, the closed-loop poles corresponding to the $n$ critical modes should be on the left side of the constant $\sigma\left(\sigma_{r e f}=-0.4\right)$ line to ensure a maximum settling time of $10.0 \mathrm{~s}$. Minimizing the 
difference between the desired $\left(\zeta_{\text {ref }}\right)$ and actual damping ratios for each inter-area modes guarantees a specified settling time without appreciable change in frequency. The third specification is taken care of by minimizing the average controller gain mean $(A)$ over the frequency range of interest $(0.3$ to $0.6 \mathrm{~Hz})$.

An objective function is formulated to include these three design specifications for $n$ critical inter-area modes under $k$ probable operating conditions. The individual objectives, (1), (2) and (3), are combined with appropriate weights $\left(\alpha_{j}\right)$ to form the overall objective function, given by (4) [18].

$$
\begin{aligned}
& f_{1}=\sum_{p=1}^{n} \sum_{q=1}^{k}\left(\sigma_{\text {ref }}-\sigma_{p q}\right)^{2} \\
& f_{2}=\sum_{p=1}^{n} \sum_{q=1}^{k}\left(\zeta_{\text {ref }}-\zeta_{p q}\right)^{2} \\
& f_{3}=\sum_{p=1}^{n} \sum_{q=1}^{k} \operatorname{mean}\left(A_{p q}\right)^{2} \\
& F=\sum_{j=1}^{3} \alpha_{j} \times f_{j}
\end{aligned}
$$

In (1) - (3), the suffixes $p$ and $q$ denotes the mode number and the operating condition number, respectively. The weights $\left(\alpha_{j}\right)$ are chosen such that contribution of individual objectives, (1), (2) and (3), based on the initial guess of parameters, is similar in the overall objective function (4). However, the selection of these weights would vary depending on the designer's preference and the corresponding objectives can be penalized accordingly.

\subsection{Constraints}

Stability of the closed-loop system and also the controller itself requires the following constraints to be included in the optimization:

- To avoid any unstable poles and zeros of the controller and also to ensure that none of the controller mode is too fast, an upper limit of $T_{\max }$ and a lower limit of $T_{\min }$ were set for each of the unknown time constants $T_{j}, j=1,2, . ., 6 n$ (optimization variables), see Fig. 3 . The gains, $K_{i}, i=1,2, . ., n$ are limited in the range $K_{\min } \leq K_{i} \leq K_{\max }$. 
- Constraint on closed-loop stability is implicitly imposed through introduction of a high penalty factor $L=10^{6}$ in the objective function if there are poles on the right half of the s-plane.

The constrained optimization problem can be mathematically expressed as (5).

$$
\begin{gathered}
\min _{K_{i}, T_{j}}(F+L), i=1,2,3, \ldots, n ; j=1,2, . ., 6 n \\
\text { s.t. } T_{\min } \leq T_{j} \leq T_{\max } \forall j=1,2, . ., 6 n ; \\
K_{\min } \leq K_{i} \leq K_{\max } \forall i=1,2, . ., n \\
L=\left\{\begin{array}{c}
0 \text { if } \zeta_{p q}>0 \\
10^{6} \text { if } \zeta_{p q} \leq 0
\end{array}\right.
\end{gathered}
$$

where, $\zeta_{p q}$ is the closed loop damping ratio with the candidate controller for the $p^{\text {th }}$ mode under $q^{\text {th }}$ operating condition.

\subsection{Controller Parameters}

Solution to the above optimization problem was sought using an evolutionary technique [18] to obtain the parameters of the POD for the SVC at Hasle. All three critical outage scenarios described in Section 2 were considered in the optimization. The reference damping ratios for each of the inter-area modes were chosen to ensure a settling time of less than $10.0 \mathrm{~s}$.

In this case study the POD controller is single-input, single-output (SISO). Hence, the optimization variables are a gain $\left(K_{1}\right)$ and six time-constants $\left(T_{1}, T_{2}, \ldots . T_{6}\right)$ for the lead-lag blocks, see Fig. 3. Solution to the multi-objective constrained optimization (5) problem resulted in the controller parameters given in Table 3 .

\section{Indirect Adaptive POD (IAPOD)}

Fig. 4 shows the block diagram of an indirect adaptive regulator. The inner loop of the regulator consists of an estimator which identifies the oscillatory behavior of the non-linear system. The outer loop updates the controller parameters based on the estimated model and computes the control action. Thus the controller parameters are not updated directly, 
rather indirectly via the estimation of the system dynamics. This results in an indirect adaptive algorithm $[14,11]$.

\subsection{Online estimation}

The system (power systems with embedded FACTS devices) behavior to be controlled can be expressed as the following auto regressive moving average (ARMA) model:

$$
y(t)=-\sum_{i=1}^{n_{a}} a_{i} y(t-i)+\sum_{i=1}^{n_{b}} b_{i} u(t-k-i)+e(t)
$$

which can be transformed in z-domain as:

$$
A(z) Y(z)=z^{-k} B(z) U(z)+E(z)
$$

where,

$$
A(z)=1+\sum_{i=1}^{n_{a}} a_{i} z^{-i}, \quad B(z)=\sum_{i=1}^{n_{b}} b_{i} z^{-i}
$$

Here, $\mathrm{k}$ is the plant delay which is 1.0 considering the presence of a zero-order hold $(\mathrm{ZoH})$ due to the digital to analog converter (DAC) at the system input. $e(t)$ is a zero mean random noise (also called innovation) with the Gaussian distribution. Kalman Filtering (KF) technique [20], which provides a unifying framework for the family of Recursive Least Squares (RLS) filters, is employed to estimate the parameters of $\hat{\theta}$ (see (9)) online. The square of the prediction error $\varepsilon(t)$ is minimized with the parameter vector $\hat{\theta}(t)$ converging asymptotically to the actual values [21]. The parameter vector and the prediction error are expressed in (9) and (10) respectively.

$$
\begin{gathered}
\hat{\theta}=\left[\hat{a}_{1}, \hat{a}_{2}, \ldots \hat{a}_{n_{a}}, \hat{b}_{1}, \ldots \hat{b}_{n_{b}}\right]^{T} \\
\varepsilon(t)=y(t)-\hat{y}(t)
\end{gathered}
$$

The predicted output is given by:

$$
\hat{y}(t)=X^{T}(t) \hat{\theta}(t-1)
$$


where, $X(t)$ is the regressor containing the past input and output samples:

$$
X(t)=\left[-y(t-1), \ldots,-y\left(t-n_{a}\right), u(t-2), \ldots u\left(t-n_{b}-1\right)\right]^{T}
$$

Different methods are proposed in literature $[14,22]$ to track sudden parameter changes in the system. This is particularly important for the application of power oscillation damping since abrupt parameter variations are expected with the occurrence of a fault followed by a line outage. To address this issue, appropriate tuning of the process noise covariance matrix $\left(R_{1}\right)$ is adopted in this paper. The standard steps for implementing the KF algorithm [23, 24] are:

Step I: Calculate the prediction error (10).

Step II: Compute the gain vector $K(t)$ :

$$
K(t)=\frac{P(t-1) X(t)}{R_{2}+X^{T}(t) P(t-1) X(t)}
$$

where, $R_{2}$ is the variance of innovations e(t).

Step III: Update the covariance matrix $P(t)$ :

$$
P(t)=\left[I-K(t) X^{T}(t)\right] P(t-1)+R_{1}
$$

here $R_{1}$ is the correlation matrix of process noise.

Step IV: Update the parameter vector $\hat{\theta}(t)$ :

$$
\hat{\theta}(t)=\hat{\theta}(t-1)+K(t) \varepsilon(t)
$$

It should be mentioned that the appropriate identification of the mode of interest is dependent on the choice of sampling frequency. The lower limit on the sampling frequency is decided by the Nyquist criterion. On the other hand, the accuracy of the identified parameters deteriorates with over-sampling [25]. A sampling interval $\left(T_{s E}\right)$ of $80 \mathrm{~ms}$ is found out to be suitable for the present application. The controller is designed online based on the certainty equivalence principle [14] described in the next section. 


\subsection{Fixed Structure Adaptive Control}

An adaptive controller with a fixed structure but time-varying coefficients is considered in this work. Based on the identified system parameters, a pole-shifting control algorithm is used to compute the controller coefficients. Let us assume that the feedback control loop has the form:

$$
U(z) F(z)=-Y(z) G(z)
$$

where

$$
G(z)=\sum_{i=0}^{n_{g}} g_{i} z^{-i}, \quad F(z)=1+\sum_{i=1}^{n_{f}} f_{i} z^{-i}
$$

It can be shown that the optimal orders of the control polynomials are related to the order of the identified system as $n_{g}=n_{b}-1$ and $n_{f}=n_{a}-1$ respectively [7]. The underlying design problem for the controller is to shift the open-loop poles radially by the pole-shifting factor $\alpha$ towards the centre of the unit circle so that the closed loop characteristic equation assumes the form:

$$
A\left(z^{-1}\right) F\left(z^{-1}\right)+B\left(z^{-1}\right) G\left(z^{-1}\right)=A\left(\alpha z^{-1}\right)
$$

The controller parameters can be derived from the above equality. Let,

$$
Z=\left[f_{1}, f_{2}, \ldots, f_{n_{f}}, g_{0}, g_{1}, \ldots, g_{n_{g}}\right]^{T}
$$

The control $\mathrm{u}(\mathrm{t})$ can then be expressed as:

$$
u(t)=\xi^{T}(t) \cdot Z
$$

where,

$$
\xi(t)=\left[-u(t-1), \ldots,-u\left(t-n_{f}\right),-y(t), \ldots,-y\left(t-n_{g}\right)\right]^{T}
$$

For the optimal controller performance, a lower magnitude of $\alpha$ is required initially to exert most of the control effort. The value of $\alpha$ is increased as the steady-state is approached. A fixed pole-shifting factor algorithm suffers due to the above mentioned reason. Hence, 
the optimum value of pole-shifting factor is determined online following the principle of minimum variance regulator. In this method the system output in the next sample is predicted from the following equation:

$$
\hat{y}(t+1)=\xi^{T}(t) \beta+b_{1} u(t, \alpha)+e(t+1)
$$

where,

$$
\beta=\left[-b_{2},-b_{3}, \ldots-b_{n_{b}}, a_{1}, a_{2}, \ldots, a_{n_{a}}\right]^{T}
$$

Thus, the optimization problem becomes:

$$
\min _{\alpha} J(t+1, \alpha)=\min _{\alpha} E\left[\hat{y}(t+1)-y_{r}(t+1)\right]^{2}
$$

subject to the constraints:

$$
\begin{aligned}
& -\frac{1}{\Lambda}(1-\sigma)<\alpha<\frac{1}{\Lambda}(1-\sigma) \\
& u_{\min }<u(t, \alpha)<u_{\max }
\end{aligned}
$$

where, $y_{r}(t+1)$ is the desired output at the next sampling instant, $\Lambda$ is the absolute value of the largest root of $A\left(z^{-1}\right), \sigma$ is a security factor to account for the inaccuracy of the estimated parameters and $u_{\max }$ and $u_{\min }$ are the maximum and minimum limits of the actuator respectively [15].

As proposed in [15], $\Lambda$ can assume negative values as well. However, the negative poleshifting factor does not carry any physical meaning. Fundamentally, with radial shift of the poles in z-domain the real part of the eigenvalues in s-domain are shifted as a logarithmic function of $\alpha$. Therefore, a negative value of the pole-shifting factor might not be explainable for a practical system. Thus, in this work the lower limit of $\alpha$ is forced to be zero.

The parameters for Kalman filter is chosen following the design guidance in [23]. A third order model is used as suggested by most of the papers in literature which ensures better pole-shifting control. The values of the parameters used for the IAPOD are listed in Table 4. 


\section{Results: Linear Analysis}

\subsection{MBPOD}

The root-locus plots in Fig. 5 capture the movement of the critical eigen values (inter-area modes) towards the left half of the complex plane with the designed MBPOD in Section 3. The feedback gain is varied between 0 (open-loop) and 1 (closed-loop) for these plots. The change of frequency from open-loop to closed-loop is very little as imposed through optimization. Besides satisfactory performance in terms of improving the damping of both inter-area modes, the robustness is also illustrated through consistent nature of the root-loci under different line outage conditions. For $6500-6700$ outage, although the movement of the 2nd $(0.55 \mathrm{~Hz})$ mode is blocked by a neighboring zero it still achieves a closed-loop damping of more than $10 \%$ which is adequate to ensure a $10 \mathrm{~s}$ settling time. None of the other modes are adversely impacted under any of the operating conditions shown.

\subsection{IAPOD}

Online estimation of power system oscillatory response and behavior of the adaptive poleshifting control is illustrated here for two outage scenarios. Convergence of estimated coefficients, $a_{i}, b_{i}$, see (6), following a disturbance (3phase fault) is shown in the upper subplot of Fig. 6. The lower subplot shows the variation of estimated frequencies. The 2nd mode $(\sim 0.5 \mathrm{~Hz})$ is picked up and damped before the 1st mode $(\sim 0.3 \mathrm{~Hz})$. Due to the chosen model order of 3 (see Table 4 and (6)) the estimator picks up only one mode at a time and damps them sequentially.

In Fig. 7, the parameter convergence is shown for a different (line 6500-6700) outage condition. Note that the parameters converge to different values as compared to those in Fig. 6 because of the change in operating condition. Also the variation of the pole-shifting factor, $\alpha$ (see (18)) computed by the self-optimizing algorithm (24), and the controller stability index are shown in the middle and lower subplots, respectively. Initially the poleshifting factor goes down to a low value to apply most of the control effort before gradually assuming higher values as the oscillations damp out. This ensures better use of control range rather than a fixed pole-shifting approach. 
The stability of the adaptive pole-shifting controller is shown in the lower subplot. The compensator becomes unstable for a moment after the fault (stability index $=1$ : unstable, 0: stable). This might be due to highly nonlinear response immediately after faults resulting in inaccurate estimation. Moreover, the recursive estimator takes some time to converge to the final value as shown in the upper subplots.

\section{Results: Non-linear Simulation}

Non-linear simulations were carried out in Matlab/Simulink to compare the performance of the MBPOD and IAPOD. Three critical tie-line outages - (i) 6500-6700 (Norwegian coast line), (ii) 7100-7000 (Finnish line) or (iii) 3359-5101 (Hasle line), see Fig. 1, were considered. It is to be noted that these three outage conditions were included in the design of the MBPOD. The performance of both MBPOD and IAPOD was tested for other scenarios (not considered in design) as well and a representative result for 3000-3115 outage is also shown.

In Figs. 8 - 11 a comparison of the dynamic behavior with no control (in green or light gray), with IAPOD (in red or gray) and with MBPOD (in blue or black) are shown. These responses are following a 3-phase short circuit near one end of the line for $80 \mathrm{~ms}$ (4-5 cycles) followed by outage of one of respective lines marked on the top of the plots.

Fig. 8 shows the power flow in lines 6500-6700 (upper subplot) and 7000-7100 (middle subplot) and the voltage (lower subplot) at the SVC near Hasle. It can be seen that the oscillation in power flows are damped satisfactorily by both the MBPOD and IAPOD. Voltage at the SVC bus shows signs of relatively high frequency variations which is possibly due to numerical solution of (24).

Fig. 9 shows the power flow in lines 3359-5101 (upper subplot) and 7000-7100 (middle subplot) and the voltage (lower subplot) at the SVC near Hasle. Although power flow oscillations settles satisfactorily with both MBPOD and IAPOD, there are larger transients for IAPOD indicating higher control effort requirement.

Fig. 10 shows the power flow in line 3359-5101 (upper subplot), the susceptance of the SVC (middle subplot) and the voltage (lower subplot) at the SVC near Hasle. As before the 
initial transients are larger with IAPOD but the damping performances are very similar.

System response following outage of line 3000-3115, not considered in the design of the MBPOD, is shown in Fig. 11. Same observation in terms of slightly larger transients for IAPOD is true for this case as the earlier ones. High frequencies in the variation of susceptance of the SVC with IAPOD is also apparent here.

To summarize, the damping performance of the IAPOD is very similar to that of a MBPOD for a wide range of operating (line outage) conditions. However, the transient behavior of SVC susceptance and voltage is slightly inferior for an IAPOD both in terms of larger and higher frequency variations.

\section{Conclusions}

A case study on the Nordic equivalent system is presented to illustrate damping of critical inter-area modes through a single static VAr compensator located in south-east Norway. Feedback signals from remote phasor measurement units (PMUs) in Norway and Finland are used. A comparison between MBPOD which is dependant on accurate system model and IAPOD which relies only on measurements is shown. The former uses an optimization approach to determine the parameters of the fixed structure controller while the latter is based on online Kalman filter estimation and adaptive pole-shifting control. It is shown that the IAPOD yields almost similar performance as MBPOD with very little prior information about the system. Although the damping performance of the IAPOD is very similar to that of a MBPOD, the transient behavior of SVC susceptance and voltage is slightly inferior for an IAPOD both in terms of larger and higher frequency variations. The performance comparison is verified for several key tie-line outages. 


\section{References}

[1] I. Kamwa, A. Heniche, G. Trudel, M. Dobrescu, R. Grondin, and D. Lefebvre, "Assessing the technical value of FACTS-based wide-area damping control loops," in proceedings of IEEE Power Engineering Society General Meeting, 2005, 2005, pp. 1734-1743 Vol. 2.

[2] J. Chow, J. Sanchez-Gasca, H. Ren, and S. Wang, "Power system damping controller design-using multiple input signals," IEEE Control Systems Magazine, vol. 20, no. 4, pp. $82-90,2000$.

[3] I. Kamwa, R. Grondin, and Y. Hebert, "Wide-area measurement based stabilizing control of large power systems-a decentralized/hierarchical approach," IEEE Transactions on Power Systems, vol. 16, no. 1, pp. 136-153, 2001.

[4] M. Aboul-Ela, A. Sallam, J. McCalley, and A. Fouad, "Damping controller design for power system oscillations using global signals," IEEE Transactions on Power Systems, vol. 11, no. 2, pp. 767-773, 1996.

[5] B. Chaudhuri and B. Pal, "Robust damping of multiple swing modes employing global stabilizing signals with a TCSC," in IEEE Power Engineering Society General Meeting, vol. 2, 2004, p. 1709 .

[6] B. C. Kuo and F. Golnaraghi, Automatic control systems, 8th ed. New York ; Chichester: Wiley, 2003.

[7] S. Cheng, Y. S. Chow, O. P. Malik, and G. S. Hope, "An adaptive synchronous machine stabilizer," IEEE Transactions on Power Systems, vol. 1, no. 3, pp. 101-109, 1986.

[8] J. Kanniah, O. Malik, and G. Hope, "Self-tuning regulator based on dual-rate sampling," IEEE Transactions on Automatic Control, vol. 29, no. 8, pp. 755-759, 1984.

[9] R. Sadikovic, P. Korba, and G. Andersson, "Self-tuning controller for damping of power system oscillations with FACTS devices," in proceedings of IEEE Power Engineering Society General Meeting, Montreal, 2006. 
[10] P. Korba, M. Larsson, B. Chaudhuri, B. Pal, R. Majumder, R. Sadikovic, and G. Andersson, "Towards real-time implementation of adaptive damping controllers for FACTS devices," in proceedings of IEEE Power Engineering Society General Meeting, Tampa, 2007.

[11] A. Domahidi, B. Chaudhuri, P. Korba, R. Majumder, and T. C. Green, "Self-tuning flexible ac transmission system controllers for power oscillation damping: a case study in real time," IET Generation, Transmission \& Distribution, vol. 3, no. 12, pp. 1079-1089, 2009.

[12] P. Li, X. Wu, C. Lu, J. Shi, J. Hu, J. He, Y. Zhao, and A. Xu, "Implementation of CSG's wide-area damping control system: Overview and experience," in IEEE PSCE '09 Power Systems Conference and Exposition, 2009, pp. 1-9.

[13] S. Cheng, O. Malik, and G. Hope, "Damping of multi-modal oscillations in power systems using a dual-rate adaptive stabilizer," IEEE Transactions on Power Systems, vol. 3, no. 1, pp. 101-108, 1988.

[14] K. J. Astrom and B. Wittenmark, Adaptive control, 2nd ed. Reading, Mass. ; Wokingham: Addison-Wesley, 1995.

[15] O. Malik, G. Chen, G. Hope, Y. Qin, and G. Xu, "Adaptive self-optimising pole shifting control algorithm," IEE Proceedings - Control Theory and Applications, vol. 139, no. 5, pp. 429-438, 1992.

[16] E. Johansson, K. Uhlen, A. B. Leirbukt, P. Korba, J. O. Gjerde, and L. K. Vormedal, "Coordinating power oscillation damping control using wide area measurements," in proceedings of IEEE/PES Power Systems Conference and Exposition, PSCE '09, 2009.

[17] S. Ray, B. Chaudhuri, and R. Majumder, "Appropriate signal selection for damping multi-modal oscillations using low order controllers," in proceedings of IEEE Power Engineering Society General Meeting, 2008, Pittsburgh, 2008. 
[18] B. Chaudhuri, S. Ray, and R. Majumder, "Robust low-order controller design for multimodal power oscillation damping using flexible AC transmission systems devices," IET Generation, Transmission \& Distribution, vol. 3, no. 5, pp. 448-459, 2009.

[19] "IEEE std 421.5 - 2005, IEEE recommended practice for excitation system models for power system stability studies," pp. 1-85, 2006.

[20] S. S. Haykin, Adaptive filter theory, 3rd ed., ser. Prentice Hall information and system sciences series. Englewood Cliffs, N.J. ; London: Prentice Hall, 1995.

[21] K. J. Astrom and B. Wittenmark, "On self tuning regulators," Automatica, vol. 9, no. 2, pp. 185-199, 1973.

[22] P. E. Wellstead and M. B. Zarrop, Self-tuning Systems. Control and Signal Processing. John Wiley \& Sons, 1991.

[23] M. Zima, M. Larsson, P. Korba, C. Rehtanz, and G. Andersson, "Design aspects for wide-area monitoring and control systems," Proceedings of the IEEE, vol. 93, no. 5, pp. 980-996, 2005.

[24] A. B. Leirbukt, J. O. Gjerde, P. Korba, K. Uhlen, L. K. Vormedal, and L. Warland, "Wide area monitoring experiences in Norway," in proceedings of IEEE PES Power Systems Conference and Exposition, PSCE '06, 2006, pp. 353-360.

[25] K. J. Astrom, "On the choice of sampling rates in parametric identification of time series," Information Sciences, vol. 1, no. 3, pp. 273-278, 1969. 
Table 1: Damping and Frequencies Under Different Scenarios

\begin{tabular}{|c||c|c||c|c|}
\hline \multicolumn{1}{|c||}{} & \multicolumn{2}{c|}{ Mode 1 } & \multicolumn{2}{c|}{ Mode 2 } \\
\hline Scenario & Damp & Freq (Hz) & Damp & Freq (Hz) \\
\hline \hline Nominal & 0.048 & 0.291 & 0.054 & 0.551 \\
\hline 3359-5101 outage & 0.049 & 0.291 & 0.029 & 0.499 \\
\hline 6500-6700 outage & 0.049 & 0.289 & 0.034 & 0.547 \\
\hline $7000-7100$ outage & 0.027 & 0.251 & 0.042 & 0.549 \\
\hline
\end{tabular}

Table 2: Selection of Feedback Signals: Magnitude and Phase Angle of Residues for Difference Between Voltage Angles measured by PMUs

\begin{tabular}{|c||c|c||c|c|}
\hline \multicolumn{1}{|c||}{} & \multicolumn{2}{c|}{ Mode 1 } & \multicolumn{2}{c|}{ Mode 2 } \\
\hline Signal & Mag & Angle (deg) & Mag & Angle (deg) \\
\hline \hline $5101-5603$ & 0.03 & -64 & 0.68 & -80 \\
\hline $5101-6100$ & 0.05 & -68 & 0.9 & -84 \\
\hline $5101-6700$ & 0.3 & 108 & 2.73 & 96 \\
\hline $5101-7000$ & 2.28 & 110 & 1.55 & 83 \\
\hline $5101-7100$ & 1.26 & 107 & 1.97 & 94 \\
\hline $5603-6100$ & 0.01 & -79 & 0.23 & -94 \\
\hline $5603-6700$ & 0.34 & 109 & 3.41 & 97 \\
\hline $5603-7000$ & 2.31 & 110 & 2.22 & 88 \\
\hline $5603-7100$ & 1.29 & 107 & 2.65 & 96 \\
\hline $6100-6700$ & 0.35 & 109 & 3.63 & 96 \\
\hline $\mathbf{6 1 0 0 - 7 0 0 0}$ & $\mathbf{2 . 3 2}$ & $\mathbf{1 1 0}$ & $\mathbf{2 . 4 4}$ & $\mathbf{8 8}$ \\
\hline $6100-7100$ & 1.31 & 107 & 2.87 & 95 \\
\hline $6700-7000$ & 1.97 & 111 & 1.26 & -67 \\
\hline $6700-7100$ & 0.96 & 107 & 0.77 & -77 \\
\hline $7000-7100$ & 1.02 & -66 & 0.53 & 127 \\
\hline
\end{tabular}

Table 3: Gains and time-constants of the MBPOD, see Fig. 3 for notations

\begin{tabular}{|c||c||c||c||c||c||c|}
\hline$K_{1}$ & $T_{1}$ & $T_{2}$ & $T_{3}$ & $T_{4}$ & $T_{5}$ & $T_{6}$ \\
\hline \hline 0.0454 & 1.3724 & 0.0100 & 0.3103 & 0.3629 & 1.6466 & 1.0571 \\
\hline
\end{tabular}

Table 4: Parameters used for the IAPOD

\begin{tabular}{|c||c||c|}
\hline Parameter & Value & Eqn. no. \\
\hline \hline$n_{a}$ & 3 & $(6)$ \\
\hline$n_{b}$ & 3 & $(6)$ \\
\hline$R_{1}$ & $\Re^{\left(n_{a}+n_{b}\right) \times\left(n_{a}+n_{b}\right)}$ & $(14)$ \\
\hline$R_{2}$ & 1.0 & $(13)$ \\
\hline$y_{r}(t+1)$ & 0.0 & $(24)$ \\
\hline$u_{\max }$ & $0.015 \mathrm{pu}$ & $(25)$ \\
\hline$u_{\min }$ & $-0.015 \mathrm{pu}$ & $(25)$ \\
\hline$T_{s E}$ & $80 \mathrm{~ms}$ & - \\
\hline$T_{s C}$ & $80 \mathrm{~ms}$ & - \\
\hline
\end{tabular}




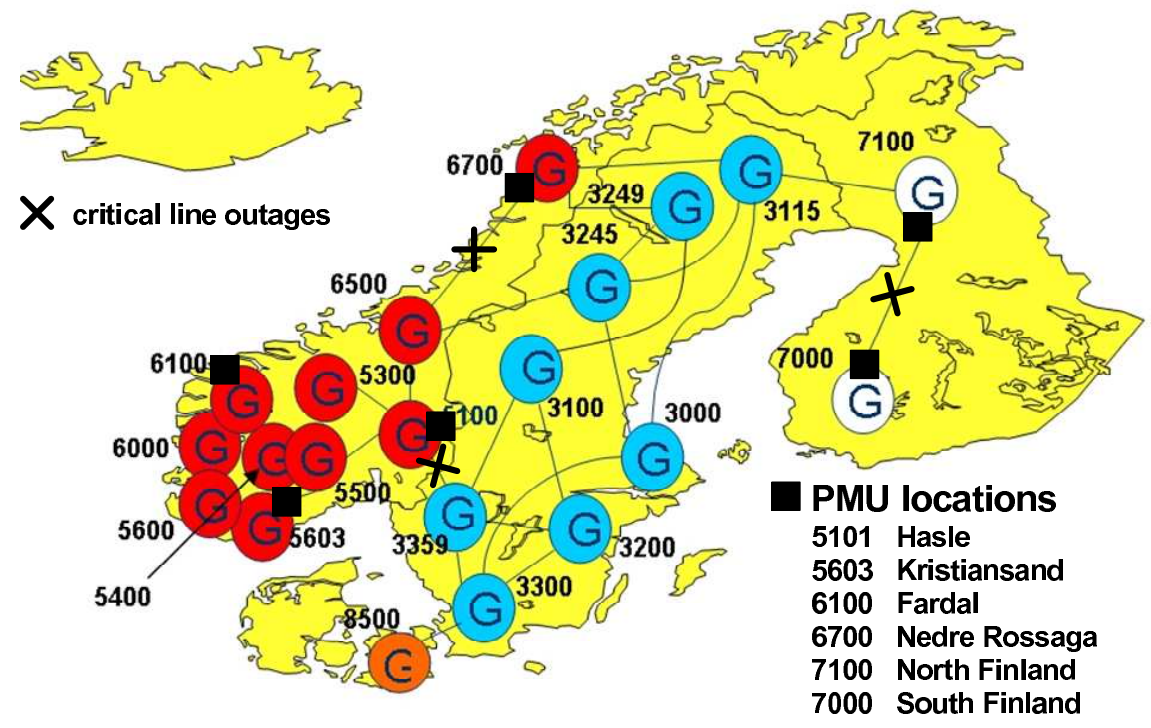

Figure 1: Nordic Equivalent System. Location of the PMUs and key tie-line outages are marked.
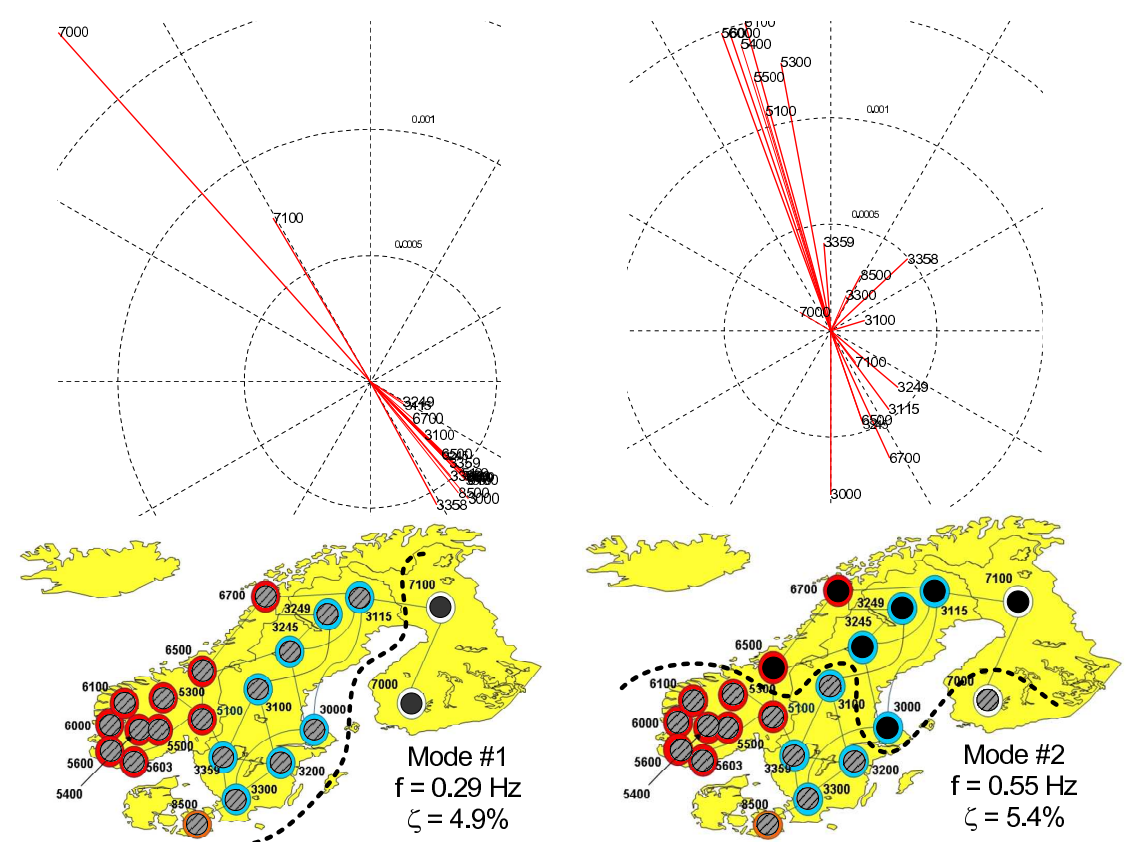

Figure 2: Modeshape and its geographical distribution for two inter-area modes. Dotted lines separate the groups of generators swinging against each other in each mode. 


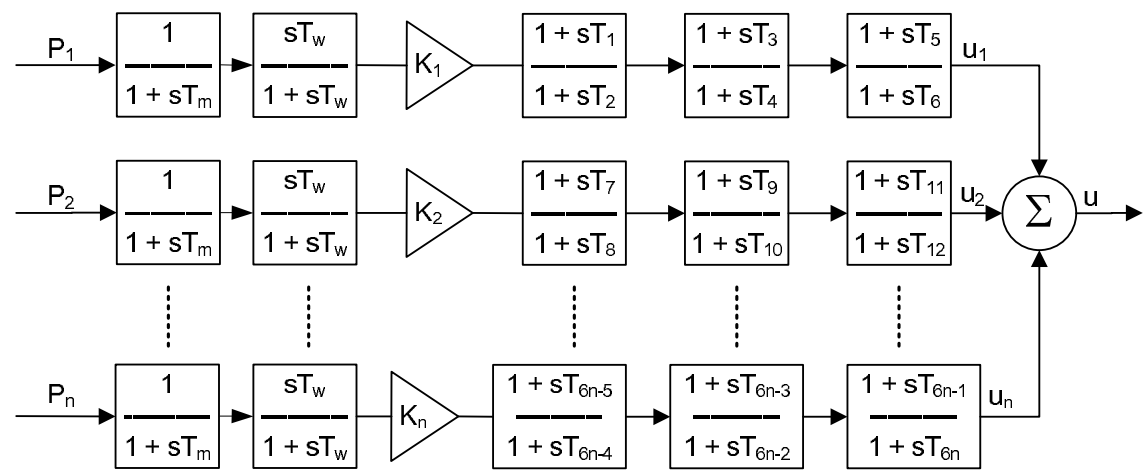

Figure 3: General block diagram of $n$-input, 1-output controller.

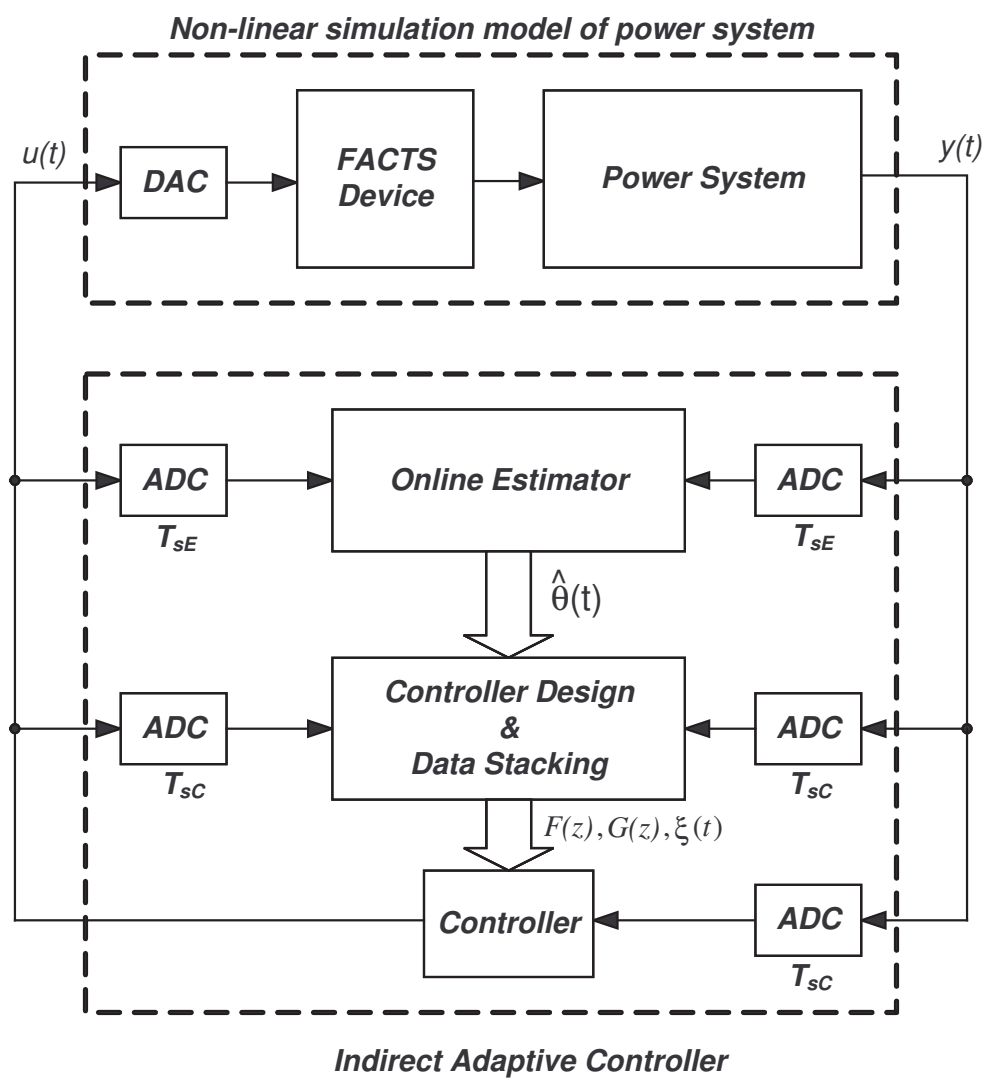

Figure 4: Overall structure of the IAPOD. 

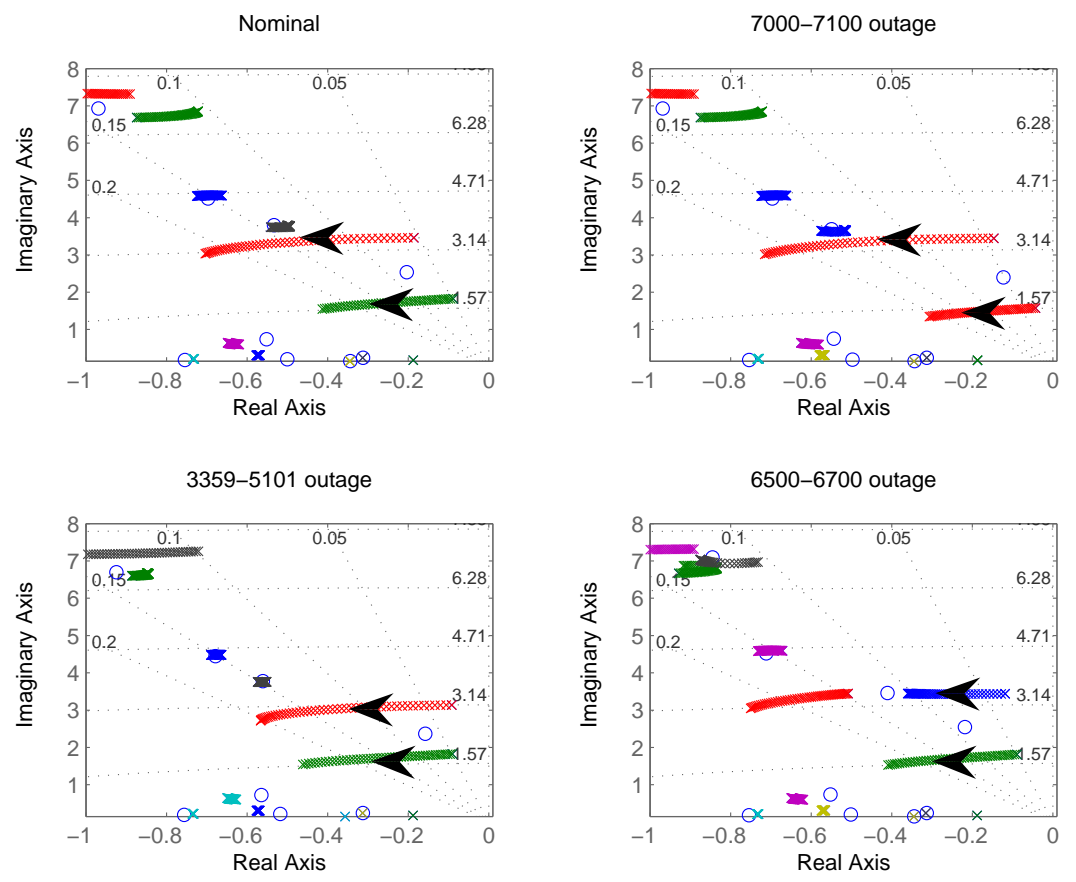

Figure 5: Root locus plots with the MBPOD under nominal and line outage scenarios.
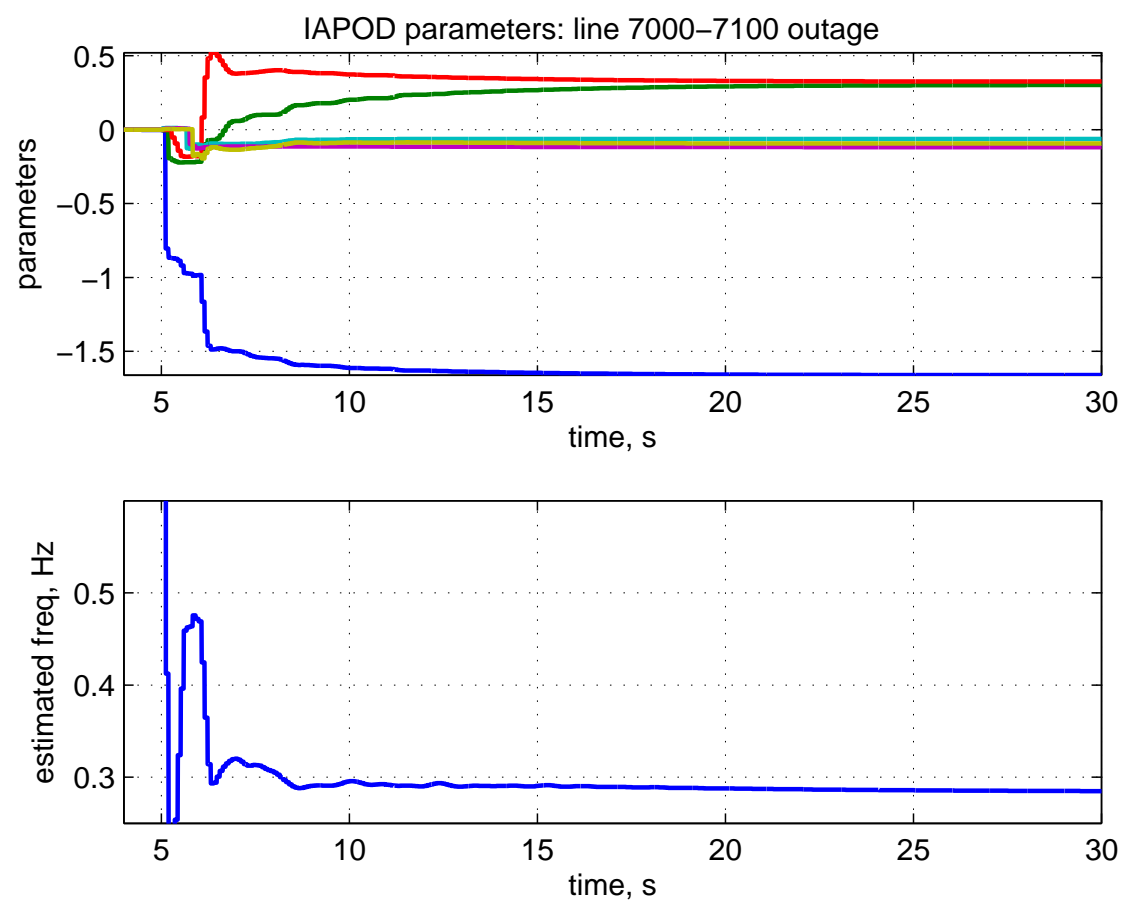

Figure 6: Behavior of IAPOD for 7000-7100 outage. 

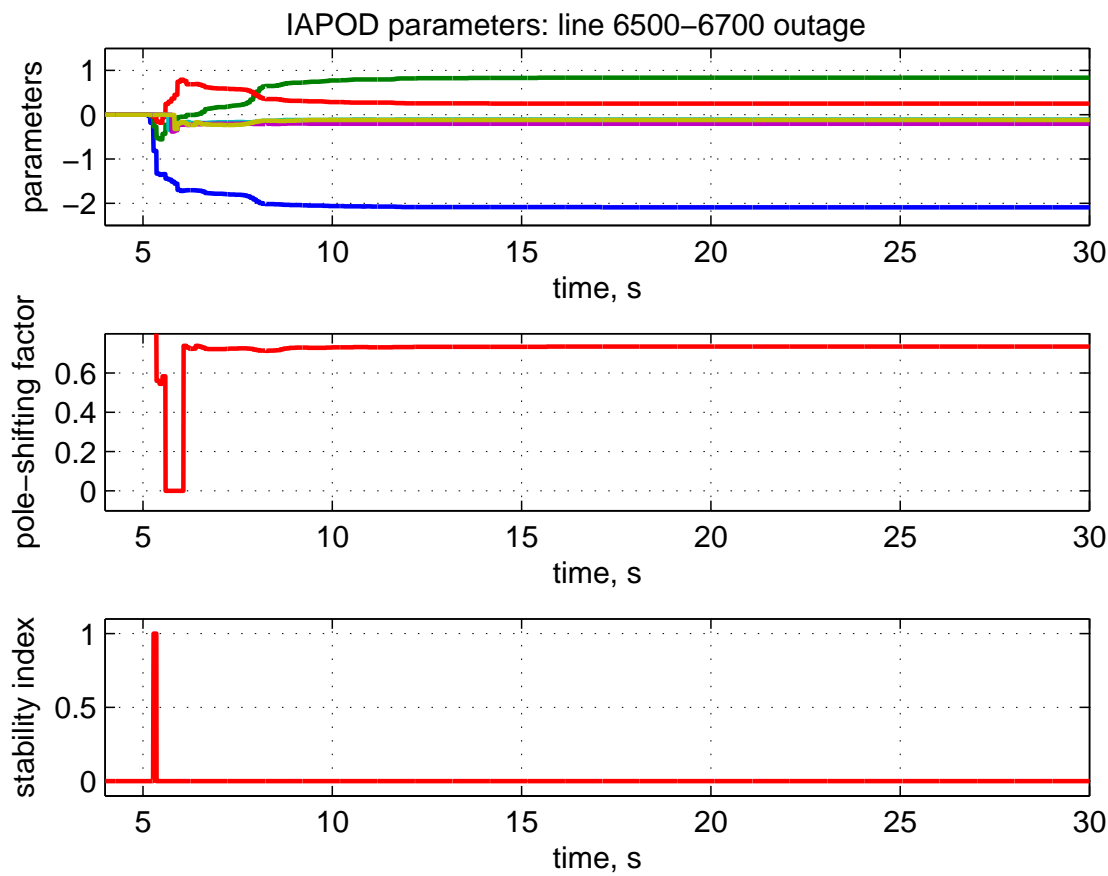

Figure 7: Behavior of IAPOD for $6500-6700$ outage. controller stability index $=1$ : unstable, 0 : stable
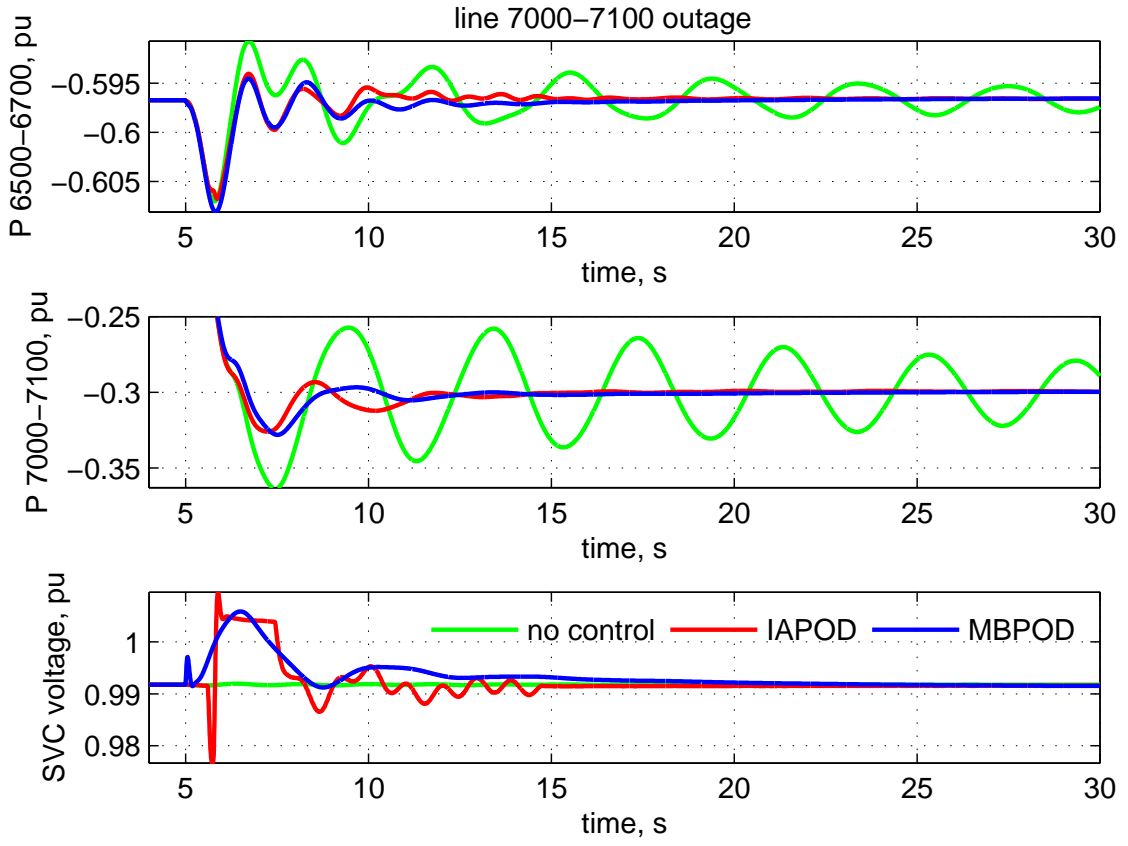

Figure 8: Dynamic response of the system following 7000-7100 outage. 

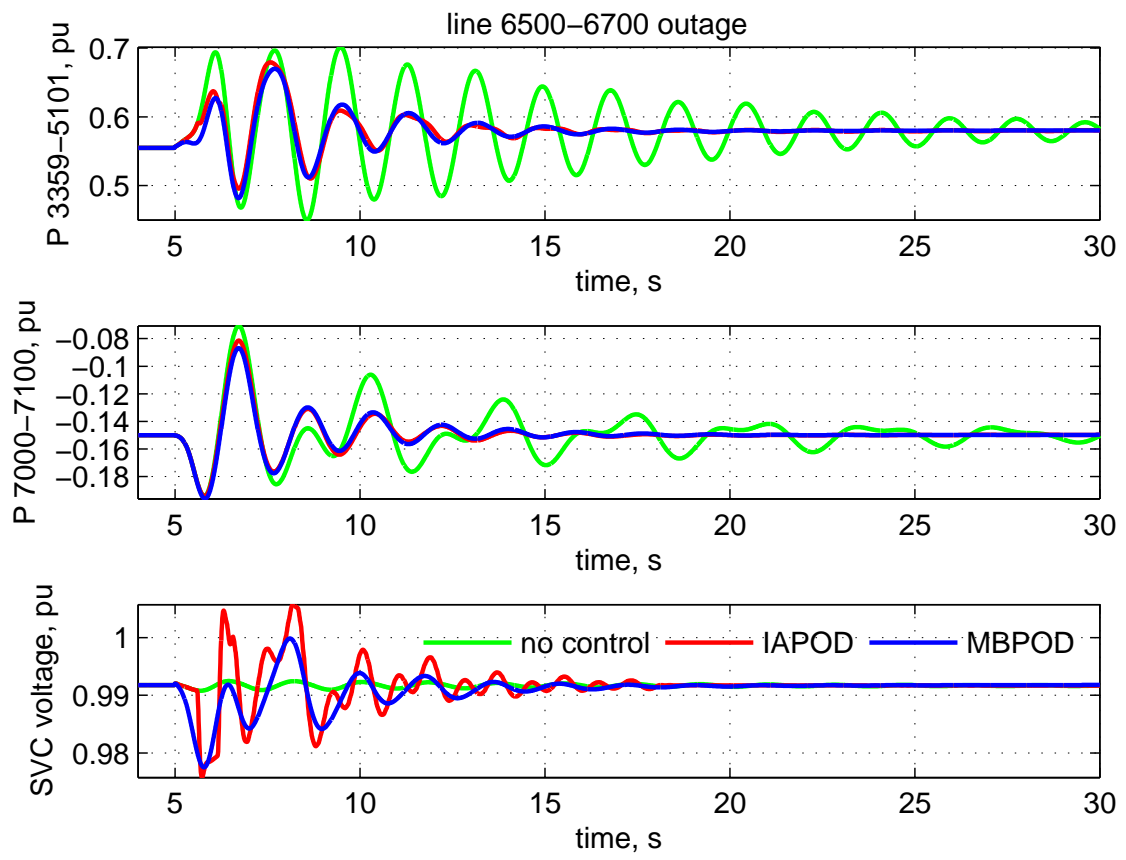

Figure 9: Dynamic response of the system following 6500-6700 outage.
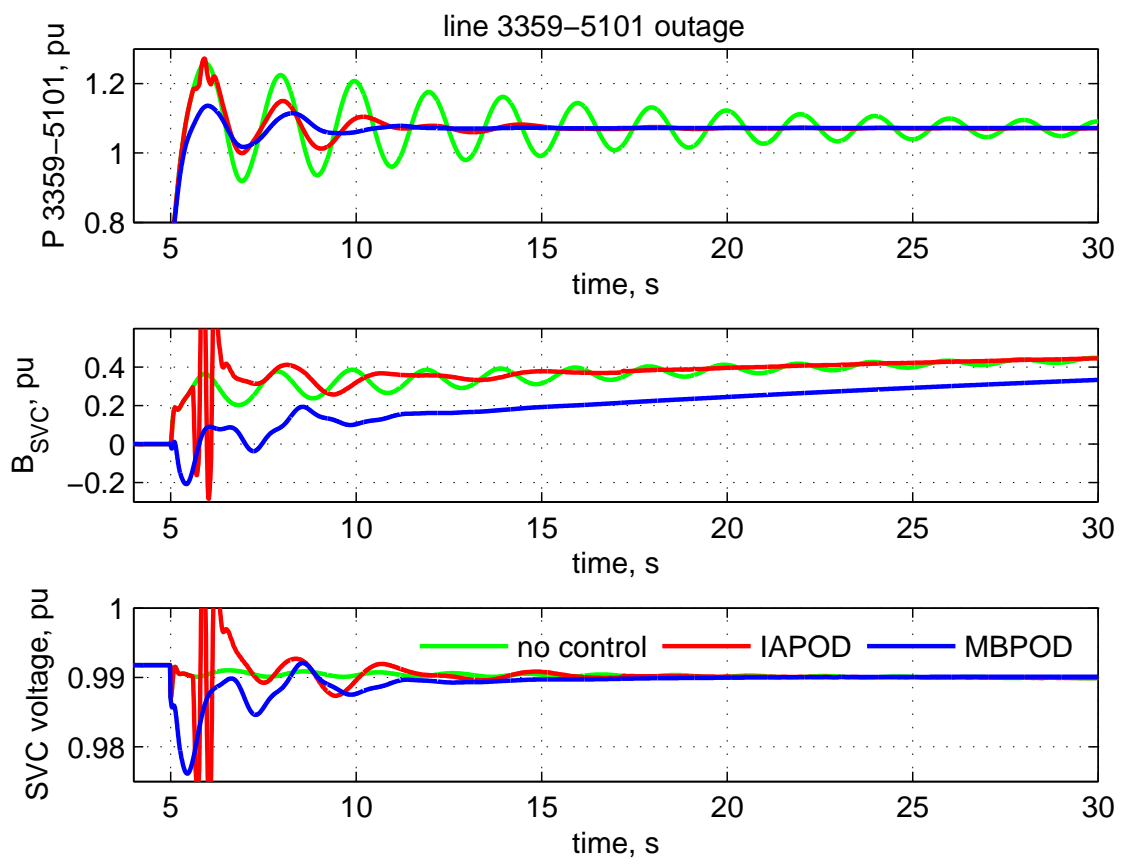

Figure 10: Dynamic response of the system following 3359-5101 outage. 

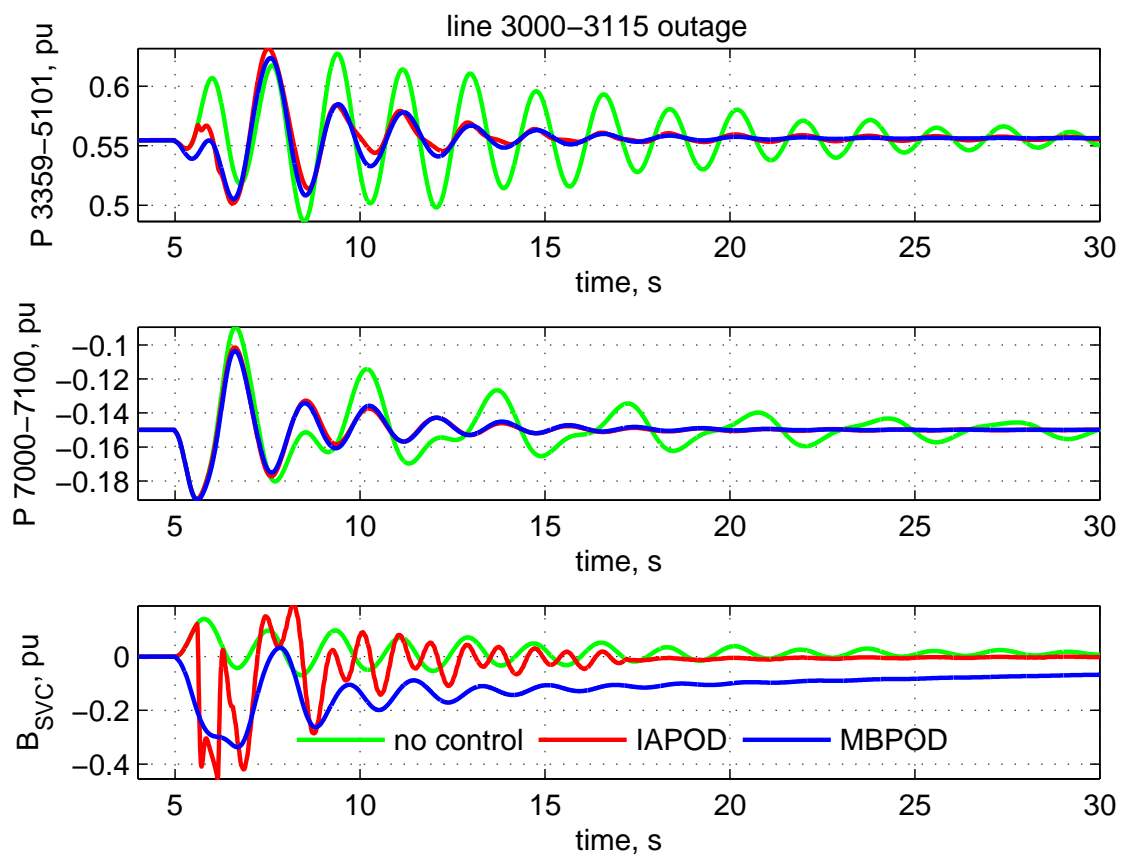

Figure 11: Dynamic response of the system following 3000-3115 outage. 